\title{
EXISTENCE OF SOLUTIONS TO AN EVOLUTION EQUATION AND A JUSTIFICATION OF THE DSM FOR EQUATIONS WITH MONOTONE OPERATORS *
}

\author{
N. S. HOANG ${ }^{\dagger}$ AND A. G. RAMM $\ddagger$
}

\begin{abstract}
An evolution equation, arising in the study of the Dynamical Systems Method (DSM) for solving equations with monotone operators, is studied in this paper. The evolution equation is a continuous analog of the regularized Newton method for solving ill-posed problems with monotone nonlinear operators $F$. Local and global existence of the unique solution to this evolution equation are proved, apparently for the first time, under only the assumption that $F^{\prime}(u)$ exists and is continuous with respect to $u$. The earlier published results required more smoothness of $F$. The Dynamical Systems Method (DSM) for solving equations $F(u)=0$ with monotone Fréchet differentiable operator $F$ is justified under the above assumption apparently for the first time.
\end{abstract}

Key words. Dynamical systems method (DSM), nonlinear operator equations, monotone operators.

AMS subject classifications. 47J05, 47J06, 47J35.

1. Introduction The Dynamical Systems Method (DSM) for solving an operator equation $F(u)=f$ in a Hilbert space consists of finding a nonlinear map $\Phi(u, t)$ such that the Cauchy problem

$$
\dot{u}=\Phi(t, u), \quad u(0)=u_{0} ; \quad \dot{u}:=\frac{d u}{d t},
$$

has a unique global solution, there exists $\lim _{t \rightarrow \infty} u(t):=u(\infty)$, and $F(u(\infty))=f$ (see [5]-[9]). Here $u_{0} \in H$ is an arbitrary element, possibly belonging to a bounded subset of $H$.

One of the versions of the DSM [5] for solving the nonlinear operator equation

$$
F(u)=f
$$

with monotone continuously Fréchet differentiable operator $F$ in a Hilbert space is based on a regularized continuous analog of the Newton method, which consists of solving the following Cauchy problem

$$
\dot{u}=-\left(F^{\prime}(u)+a(t) I\right)^{-1}(F(u)+a(t) u-f), \quad u(0)=u_{0} .
$$

Here $F: H \rightarrow H$ is a monotone continuously Fréchet differentiable operator in a Hilbert space $H, u_{0}$ and $f$ in $H$ are arbitrary, and $a(t)>0$ is a continuously differentiable function, defined for all $t \geq 0$ and decaying to zero as $t \rightarrow \infty$. This function is a regularizing function: if $F^{\prime}(u)$ is not a boundedly invertible operator and $F$ is monotone then $F^{\prime}(u) \geq 0$ and the operator $F^{\prime}(u)+a(t) I$ is boundedly invertible if $a(t)>0$.

*Received: August 28, 2009; accepted (in revised version): November 6, 2009. Communicated by Shi Jin.

${ }^{\dagger}$ Mathematics Department, Kansas State University, Manhattan, KS 66506-2602, USA, (nguyenhs@math.ksu.edu).

${ }_{\ddagger}$ Mathematics Department, Kansas State University, Manhattan, KS 66506-2602, USA, (ramm@math.ksu.edu). 
Throughout this paper we denote $I$ to be the identity operator, $\langle u, v\rangle$ to be the inner product in $H, y$ to be the minimal-norm solution to (1.1), and $c>0$ to be various estimation constants.

If $F$ is monotone and continuous, then the minimal-norm solution to (1.1) exists and is unique (see, e.g., [5]). Monotonicity of $F$ is understood as follows

$$
\langle F(u)-F(w), u-w\rangle \geq 0, \quad \forall u, w \in H .
$$

The DSM is a basis for developing efficient numerical methods for solving operator equations, both linear and nonlinear, especially when the problems are ill-posed, for example, when $F^{\prime}(u)$ is not a boundedly invertible operator (see $[5,2,3]$ ).

If one has a general evolution problem with a nonlinear operator in a Hilbert (or Banach) space

$$
\dot{u}=B(u), \quad u(0)=u_{0},
$$

then the local existence of the solution to this problem is usually established by assuming that $B(u)$ satisfies a Lipschitz condition, and the global existence is usually established by proving a uniform bound on the solution:

$$
\sup _{t \geq 0}\|u(t)\|<c
$$

where $c>0$ is a constant.

In (1.2) the operator

$$
B(u)=-\left(F^{\prime}(u)+a(t) I\right)^{-1}(F(u)+a(t) u-f)
$$

is Lipschitz if one assumes that

$$
\sup _{\left\{u:\left\|u-u_{0}\right\| \leq R\right\}}\left\|F^{(j)}(u)\right\| \leq M_{j}(R), \quad 0 \leq j \leq 2 .
$$

This assumption was used in many cases in [5] and a bound (1.5) was established under suitable assumptions in [5].

There are many results (see, e.g., [1, 4] and references therein) concerning the properties and global existence of the solution to (1.4) if $-B(u)$ is a maximal monotone operator. However, even when $F$ is a monotone operator, the operator $-B$ in the right-hand side of (1.2) is not monotone. Therefore these known results are not applicable. Even the proof of local existence is an open problem if one makes only the following assumption:

Assumption A:

$F$ is monotone and $F^{\prime}(u)$ is continuous with respect to $u$.

The main result of this paper is a proof, apparently published for the first time, that under Assumption A problem (1.2) has a unique local solution $u(t)$, and that under assumptions (2.2) on $a(t)$ (see below) this local solution exists for all $t \geq 0$, so it is a global solution. These results are formulated in Theorems 1.1 and 2.1.

Moreover, if the equation $F(y)=f$ has a solution and $y$ is its (unique) minimalnorm solution, and if $\lim _{t \rightarrow \infty} a(t)=0$ and $\lim _{t \rightarrow \infty} \frac{\dot{a}(t)}{a(t)}=0$, then there exists $u(\infty)$, and $u(\infty)=y$. This justifies the DSM for solving the equation $F(u)=0$ with a monotone continuously Fréchet differentiable operator $F$, for the first time under the weak Assumption A. This result is formulated in Theorem 3.1. 
Let us prove the existence of the solution to (1.2).

Let

$$
\psi(t)=F(u)+a(t) u-f:=\Psi(u, t):=\Psi(u) .
$$

If $a(t)>0$ and $F$ is monotone and hemicontinuous, then it is known (see, e.g., [1, p. $100])$ that the operator $F(u)+a(t) u$ is surjective. If $F^{\prime}(u)$ is continuous, then, clearly, $F$ is hemicontinuous. If $F$ is monotone and $a(t)>0$ then, clearly, the operator $F(u)+$ $a(t) u$ is injective. Thus, Assumption A implies that the operator $F(u)+a(t) u$ is injective and surjective, it is continuously Fréchet differentiable, as well as its inverse, so the map $u \mapsto F(u)+a(t) u$ is a diffeomorphism. Therefore equation (1.6) is uniquely solvable for $u$ for any $\psi$, and the inverse map $\psi=\Psi(u)$ is a diffeomorphism. The inverse map $u=U(\psi)$, is continuously differentiable by the inverse function theorem since the operator $\Psi_{u}^{\prime}=F^{\prime}(u)+a(t) I$ is boundedly invertible if $a(t)>0$. Recall that $F^{\prime}(u) \geq 0$, because $F$ is monotone. If $a(t) \in C^{1}([0, \infty))$ then the solution $u=u(t)$ of equation (1.6) is continuously differentiable with respect to $t$ (see [5, pp. 260-261]), and if $u=u(t)$ is continuously differentiable with respect to $t$, then so is $\psi(t)=\Psi(u(t))$. The differentiability of $u(t)=U(\psi(t))$ also follows from a consequence of the classical inverse function theorem (see, e.g., [1, Corollary 15.1, p. 150]). Therefore, equation (1.2) can be written in an equivalent form as

$$
\dot{\psi}(t)=\dot{a}(t) u(t)-\psi(t):=Q(t, \psi), \quad \psi(0):=\psi\left(u_{0}\right),
$$

where $u(t)=U(\psi(t))$ is continuously differentiable with respect to $t$ and $\psi(t)$ is continuously differentiable with respect to $t$. The map $Q(t, \psi)$ is Lipschitz with respect to $\psi$, and the local existence of the solution to problem (1.7) follows from the standard result (see, e.g., [5, p.247]). Since the map $U(\psi)$ is continuously differentiable and $\dot{\psi}$ is a continuous function of $t$, the function $\dot{u}$ is a continuous function of $t$, and problem (1.7) is equivalent to problem (1.2). We have proved the following theorem.

THEOREM 1.1. If Assumption A holds, then problem (1.2) has a unique local solution.

In Section 2 we discuss existence of the global solution to problem (1.2).

\section{Existence of the global solution}

Since $G(t, \psi)$ is Lipschitz with respect to $\psi$ and continuously differentiable with respect to $t$, the solution to (1.7) exists globally, i.e., for all $t \geq 0$, if

$$
\sup _{t \geq 0}\|\psi(t)\| \leq c<\infty .
$$

If the solution $\psi$ to problem (1.7) exists globally, then the solution $u(t)$ to the equivalent problem (1.2) exists globally because the map $\psi \mapsto u$ is a diffeomorphism for $t \in[0, T]$, where $T>0$ is an arbitrary large number.

Let us prove (2.1) assuming that

$$
\frac{|\dot{a}(t)|}{a(t)}<\frac{1}{2}, \quad t \geq 0
$$

Denote $h(t):=\|\psi(t)\|$. We multiply both sides of (1.7) with $\psi(t)$ and obtain

$$
h \dot{h}=-h^{2}+\langle\dot{a}(t) u(t), \psi\rangle .
$$

Let $w(t)$ solve the equation

$$
F(w(t))+a(t) w(t)-f=0, \quad t \geq 0 .
$$


It is known (see [5, p.112]) that if equation (1.1) is solvable and $\lim _{t \rightarrow \infty} a(t)=0$, then $w(\infty)$ exists, and $w(\infty)=y$. So

$$
\|w(t)\|<c \quad \forall t \geq 0 .
$$

Equation (2.3) implies

$$
\dot{h} \leq-h+\|\dot{a}|\|u(t)-w(t)\|+| \dot{a}(t) \mid\| w(t) \| .
$$

Later we will prove the estimate

$$
\|u(t)-w(t)\| \leq \frac{h(t)}{a(t)}, \quad \forall t \geq 0 .
$$

If (2.6) holds, then (2.5) implies

$$
\dot{h} \leq-h\left(1-\frac{|\dot{a}(t)|}{a(t)}\right)+|\dot{a}||| w(t) \| \leq-\frac{h}{2}+c|\dot{a}(t)| .
$$

Therefore,

$$
h(t) \leq h(0) e^{-\frac{t}{2}}+c e^{-\frac{t}{2}} \int_{0}^{t} e^{\frac{s}{2}}|\dot{a}(s)| d s, \quad \forall t \geq 0 .
$$

From (2.8) and (2.2) one obtains

$$
h(t) \leq h(0) e^{-\frac{t}{2}}+c e^{-\frac{t}{2}} \int_{0}^{t} e^{\frac{s}{2}} \frac{a(s)}{2} d s, \quad \forall t \geq 0 .
$$

Since we have assumed that $a(t)>0$ is a $C^{1}([0, \infty))$ function, such that $a(t) \rightarrow 0$ as $t \rightarrow \infty$, we have $\sup _{t \geq 0} a(t)<c$. Thus, from inequality (2.9) one obtains

$$
h(t) \leq h(0) e^{-\frac{t}{2}}+c\left(1-e^{-\frac{t}{2}}\right), \quad \forall t \geq 0 .
$$

Therefore, estimate (2.1) is proved as soon as (2.6) is verified.

Let us state our result and then prove (2.6).

Theorem 2.1. If Assumption A and (2.2) hold, then problem (1.2) has a unique global solution.

Let us verify (2.6).

Using (1.3) one obtains

$$
\langle F(u)-F(w)+a(u-w), u-w\rangle \geq a\|u-w\|^{2} .
$$

Thus,

$$
\|u(t)-w(t)\| \leq \frac{\| F(u(t))-F(w(t))+a(t)(u(t)-w(t) \|}{a(t)}=\frac{h(t)}{a(t)} .
$$

So (2.6) is verified and Theorem 2.1 is proved. 


\section{Justification of the DSM}

By the justification of the DSM for solving equation

$$
F(y)=f,
$$

we mean the proof of the following statements (see [5, p. 1, formulas (1.1.5)]):

$$
\exists ! u(t), \quad \forall t \geq 0 ; \quad \exists u(\infty) ; \quad F(u(\infty))=f .
$$

In Theorem 2.1 the first of these statements is proved. Let us assume

$$
\lim _{t \rightarrow \infty} a(t)=0, \quad \lim _{t \rightarrow \infty} \frac{\dot{a}(t)}{a(t)}=0,
$$

and prove the remaining two statements from (3.2).

REMARK 3.1. Actually our argument allows for the following generalization of the results: assumption (2.2) can be weakened to $\frac{|\dot{a}(t)|}{a(t)} \leq q, \forall t \geq 0, q \in(0,1)$ and the second assumption (3.3) can be weakened to $\limsup _{t \rightarrow \infty} \frac{|\dot{a}(t)|}{a(t)} \leq q^{\prime}$, where $q+q^{\prime}<1$.

THEOREM 3.1. If Assumption A, (2.2) and (3.3) hold, and equation (3.1) has a solution, then (3.2) hold, and $u(\infty)=y$, where $y$ is the unique minimal-norm solution to (3.1).

Proof. It is known (see, [5, p.112]) that

$$
\lim _{t \rightarrow \infty} w(t)=y,
$$

so $\limsup _{t \rightarrow \infty}\|w(t)\|<c$. Inequality (2.6) implies

$$
a(t)\|u(t)\| \leq a(t)\|w(t)\|+h(t) \leq c a(t)+h(t) .
$$

Inequalities (3.5) and (2.3) imply

$$
\dot{h} \leq-h+\frac{|\dot{a}(t)|}{a(t)}[c a(t)+h(t)] .
$$

Assumptions (2.2) and (3.3) imply that

$$
\lim _{t \rightarrow \infty}|\dot{a}(t)|=0 .
$$

From the second assumption (3.3) it follows that

$$
\frac{|\dot{a}(t)|}{a(t)}<\delta, \quad \forall t>t_{\delta},
$$

where $\delta>0$ is an arbitrary small fixed number. From (3.6), (3.7), and (3.8) it follows that

$$
\lim _{t \rightarrow \infty} \psi(t)=0
$$

Indeed, (3.6) implies

$$
\dot{h} \leq-(1-\delta) h+c|\dot{a}(t)|, \quad t>t_{\delta} .
$$


Thus

$$
h(t) \leq h\left(t_{\delta}\right) e^{-(1-\delta)\left(t-t_{\delta}\right)}+c e^{-(1-\delta) t} \int_{t_{\delta}}^{t} e^{(1-\delta) s}|\dot{a}(s)| d s, \quad t \geq t_{\delta} .
$$

Clearly $\lim _{t \rightarrow \infty} h\left(t_{\delta}\right) e^{-(1-\delta) t}=0$. The L'Hospital rule yields

$$
0 \leq \lim _{t \rightarrow \infty} \frac{\int_{t_{\delta}}^{t} e^{(1-\delta) s}|\dot{a}(s)| d s}{e^{(1-\delta) t}}=\lim _{t \rightarrow \infty}(1-\delta)^{-1}|\dot{a}(t)|=0 .
$$

Thus, (3.9) is proved.

Let us prove that (3.9) implies the existence of the limit $u(\infty):=\lim _{t \rightarrow \infty} u(t)$, the relation

$$
F(u(\infty))=f
$$

and the relation $u(\infty)=y$, where $y$ is the minimal-norm solution of the equation $F(u)=f$.

It is proved in [5, p.112], that the limit $w(\infty)$, as $a=a(t) \rightarrow 0$, i.e., $t \rightarrow \infty$, of the solution $w_{a}$ to the following equation:

$$
F\left(w_{a}\right)+a w_{a}-f=0, \quad a>0,
$$

with a hemicontinuous monotone operator $F$, exists and $w(\infty)=y$, provided that equation (3.1) is solvable.

Thus, the existence of $u(\infty)$ follows from (2.6) if one proves that

$$
\lim _{t \rightarrow \infty} \frac{h(t)}{a(t)}=0
$$

To verify (3.15), we claim that the second assumption (2.2) implies that

$$
\lim _{t \rightarrow \infty} \frac{e^{-(1-\delta) t}}{a(t)}=0
$$

Indeed, the inequality $\dot{a}(t) \geq-0.5 a(t)$ implies $a(t) \geq c e^{-0.5 t}$, where $c>0$ is a constant. Thus, the claim follows if $\delta<0.5$.

Let us now prove (3.15). Divide both side of (3.11) by $a(t)$ and let $t \rightarrow \infty$. The first term on the right tends to zero, and the second term by the L'Hospital's rule tends also to zero because of the second assumption (3.3). Thus, (3.15) is established.

Since the limit $w(\infty)=y$ exists, it follows from (2.6) and (3.15) that $u(\infty)$ exists and $u(\infty)=y$.

Theorem 3.1 is proved.

\section{REFERENCES}

[1] K. Deimling, Nonlinear Functional Analysis, Springer Verlag, Berlin, 1985.

[2] N.S. Hoang and A. G. Ramm, A discrepancy principle for equations with monotone continuous operators, Nonlinear Analysis Series A: Theory, Methods \& Applications, 70, (2009), 43074315.

[3] N.S. Hoang and A. G. Ramm, Dynamical systems method for solving nonlinear equations with monotone operators, Math. of Comput., 79, (2010), 239-258.

[4] I. Miyadera, Nonlinear Semigroups, Amer.Math. Soc., Providence, RI, 1992. 
[5] A. G. Ramm, Dynamical Systems Method for Solving Operator Equations, Elsevier, Amsterdam, 2007.

[6] A. G. Ramm, Dynamical Systems Method for solving operator equations, Commun. in Nonlinear Sci. and Numer. Simulation, 9, N2, (2004), 383-402.

[7] A. G. Ramm, Dynamical Systems Method (DSM) and nonlinear problems, in the book: Spectral Theory and Nonlinear Analysis, ed J. Lopez-Gomez, World Scientific Publishers, Singapore, 2005, 201-228.

[8] A. G. Ramm, Dynamical systems method and a homeomorphism theorem, Amer. Math. Monthly, 113, N10, (2006), 928-933.

[9] A. G. Ramm, Dynamical systems method for nonlinear equations in Banach spaces, Commun. in Nonlinear Sci. and Numer. Simulation, 11, N3, (2006), 306-310. 